\title{
Effets de rations à base de déchets de mangue sur les performances pondérales et la qualité de la carcasse de porcs Korhogo en croissance au Burkina Faso
}

\author{
Timbilfou KIENDREBEOGO1*, Youssouf MOPATÉ LOGTÉNÉ2 et Chantal-Yvette KABORÉ- \\ ZOUNGRANA ${ }^{3}$ \\ 1 Centre National de la recherche Scientifique et Technologique/Institut de l'Environnement et de Recherches \\ Agricoles (INERA), Station de Farako-bâ, Bobo-Dioulasso (Burkina Faso) \\ 2 Institut de Recherche en Élevage pour le Développement (IRED) N'Djaména, Tchad, Chercheur associé au \\ LERNSE de l'Université Nazi Boni (Bobo-Dioulasso, Burkina Faso) ; \\ 3 Laboratoire d'Études et de Recherches des Ressources Naturelles et des Sciences de l'Environnement (LERNSE), \\ Université Nazi Boni (UNB) de Bobo-Dioulasso, Burkina Faso. \\ *Auteur pour la correspondance :'Téléphone : +226 7022 30 03, E-mail : timbilfou@gmail.com \\ Original submitted in on 31st May 2018. Published online at www.m.elewa.org on $30^{\text {th }}$ September 2018 \\ http://dx.doi.org/10.4314/jab.v129i1.7
}

\section{RESUME}

Objectif: L'objet de l'étude a été d'évaluer les effets de l'utilisation de rations à base de provendes de mangue sur les performances de croissance et la qualité de la carcasse de porcs en croissance $(25-60 \mathrm{~kg})$. Méthodologie et résultats : Deux régimes mangue (R1 et $R 2)$ et un témoin (R3) ont été servis à 3 lots de 8 porcs chacun. A la fin de l'essai 2 porcs /lot ont été abattus pour apprécier la qualité des carcasses. Le GMQ de R3 $(459 \pm 62 \mathrm{~g})$ a été significativement supérieur à ceux de R1 $(399 \pm 81 \mathrm{~g})$ et R2 $(412 \pm 81 \mathrm{~g})$ $(P<0,05)$. L'IC de R3 $(1,9 \pm 0.4)$ a été significativement supérieur à ceux IC de R1 $(2,5 \pm 05)$ et $R 2(0,6 \pm 06)$ $(P<0,05)$. Les rendements carcasses de R1 $(83,4 \%)$ et $R 2(77,6 \%)$ ont été plus élevés à celui de R3 $(76 \%)$. Le taux de gras de R3 (32,8\%) a été plus élevé que ceux de R1 (30,7\%) et R2 (31,7\%).

Conclusion et application : L'étude a montré l'utilisation possible des provendes de mangue en substitution au maïs. Ceci ouvre des perspectives d'accroissement de la disponibilité d'aliments pour les porcs moins compétitifs entre l'homme et les animaux. Les rations qui les incorporent sont propices à la production de viande maigre très prisée par les consommateurs. Des provendes et des formules les incorporant pourront être proposées pour l'alimentation des porcs, l'amélioration de la productivité des élevages, de la qualité des carcasses et du revenu des éleveurs. Des études complémentaires sur la digestibilité des provendes et de meilleures formules seront nécessaires pour affiner les recommandations d'utilisation aux éleveurs.

Mots clés : Rations de mangue, Porcs en croissance, Performances pondérales, Qualité des Carcasses, Burkina Faso

\footnotetext{
ABSTRACT

Objective: The purpose of the study was to evaluate the effects of the use of mango feed rations on growth performance and carcass quality of growing pigs $(25-60 \mathrm{~kg})$.

Methodology and results: Two mango diets (R1 and R2) and a control (R3) were served to 3 batches of 8 pigs each. At the end of the trial 2 pigs / lot were slaughtered to assess the quality of the carcasses. The
} 
Daily Weight Gain (DWG) of R3 $(459 \pm 62 \mathrm{~g})$ was significantly higher than those of R1 were $(399 \pm 81 \mathrm{~g})$ and R2 $(412 \pm 81 \mathrm{~g})(\mathrm{p}<0.05)$. The R3 $\mathrm{Cl}(1.9 \pm 0.4)$ was significantly $<$ at R1 $(2.5 \pm 0.5)$ and $\mathrm{R} 2(2.6 \pm 06)(p<0.05)$. Carcass yields of R1 $(83.4 \%)$ and R2 $(77.6 \%)$ were higher than that of R3 $(76 \%)$. The fat content of R3 (32.8\%) was higher than those of R1 were (30\%) and R2 (31.7\%).

Conclusion and application: The study showed the possible use of mango feed to substitute maize. This opens up prospects for increasing the availability of food and thus less competition between humans and animals. It has also shown the tendency of mango diets to produce lean meat; which is very popular to consumers. Feed and food formulas incorporating mango waste may be proposed for the feeding of pigs hence improving the productivity of livestock, the quality of carcasses and the income of farmers. Further studies on a digestibility of feed and the better diets will be needed to refine the recommendations of use to breeders.

Keywords: Mango diets, growing pigs, weight performance, carcass quality, Burkina Faso

\section{INTRODUCTION}

Sous l'effet conjugué de l'urbanisation, de la croissance démographique et des nouvelles attentes socioculturelles des populations, on assiste, dans les pays du Sud, à l'émergence d'une demande croissante en produits d'origine animale, concentrée surtout dans les villes (Mankor, 2009). Or, bien que les pays de l'Afrique de l'Ouest détiennent $25 \%$ du cheptel des pays de I'Afrique au Sud du Sahara (ASS), l'élevage est encore peu productif. Ceci se traduit par une faible consommation en protéine animale de 8,5 à $9 \mathrm{~g}$ par habitant par jour contre une recommandation de $20 \mathrm{~g}$ et un accroissement important des importations en lait et produits laitiers (FAO, 2018). Au Burkina Faso l'élevage porcin est une activité importante qui se présente comme une alternative à une rapide production de viande en réponse à une demande en forte croissance. En 2009 les effectifs porcins du Burkina Faso de 2125000 têtes (MRA, 2015) représentaient 58,09\% du cheptel des pays de l'Union Monétaire Ouest Africaine(UEMOA) qui étaient de 4159210 têtes (FAO, 2017). C'est un élevage en forte croissance numérique de 18,6\%/an (Kiendrébéogo et al., 2012a) calculé à partir des effectifs du cheptel en 1993 (660 582 têtes) et en 2003 (1 886234 têtes) contre un taux moyen de $1,34 \%$ pour les ruminants (bovins, ovins et caprins) (MED/MRA, 2004). La production porcine est une activité prépondérante à Bobo-Dioulasso (435543 habitants au dernier recensement en 2006) (MF, 2009), 2ème ville après Ouagadougou (1475839 habitants) la capitale. La région des Haut-Bassins dont Bobo-Dioulasso est la capitale comptait 212000 têtes de porcs au dernier recensement des effectifs du cheptel national et se classait en 4ème position parmi les 13 régions administratives du Burkina Faso. La contribution de l'élevage porcin en viande produite à l'Abattoir Frigorifique de Bobo-Dioulasso (AFB) a été de 10,45\% entre 2001 et 2006 (Kiendrébéogo et al., 2012a). En dépit de l'importance de l'élevage porcin largement conduit suivant le système extensif dans lequel la race locale est plus présente comparativement aux systèmes intensifiés où sont surtout élevés les races exotiques Korhogo et Large White (Kiendrébéogo et al., 2014), force est de constater que sa productivité est encore faible. En effet l'élevage porcin extensif encore dominant $195 \%$ des élevages) fourni $85 \%$ des porcs abattus à l'Abattoir Frigorifique de Bobo-Dioulasso (AFB) mais de faille poids vif moyen de $60,7 \pm 26,5 \mathrm{~kg}$ contre $90 \mathrm{~kg}$ pour les élevages intensifiés mais obtenus tardivement entre 11 et 13 mois d'âge (Kiendrébéogo et al., 2008; Kiendrébéogo et al., 2012a; Kiendrébéogo et al., 2012b). L'alimentation est la principale contrainte incriminée dans la faible productivité des élevages porcins de la zone de Bobo-Dioulasso (Kiendrébéogo et al., 2014). Elle se traduit par la faible disponibilité des aliments aggravée par la compétition entre l'homme et les monogastriques (porcs et volailles) autour des céréales, leurs coûts élevés et les mauvaises pratiques d'alimentation des porcs (Kiendrébéogo et al., 2008). L'utilisation des aliments non conventionnels à savoir les 
résidus de ménage, certaines espèces fourragères, les résidus de cultures maraîchères et les fruits dont les mangues avariées est constatée au niveau des élevages urbains et périurbains de Bobo-Dioulasso (Kiendrébéogo, 2006 ; Mopaté et al., 2011). D'énormes volumes de mangues infestées par les mouches des fruits sont toujours perdus dans les vergers, sur les lieux de transformation et dans les circuits de commercialisation (vente locale et exportation). Très peu de travaux et d'informations sont disponibles sur la valorisation des sous-produits de la mangue pour alimenter les animaux. Toutefois, des travaux postérieurs à la présente étude ont concerné la croissance de poulets de race locale (Sawadogo, 2016), la croissance et la ponte

\section{MATERIEL ET METHODES}

Site de l'étude : Le test de rationnement a été conduit dans une unité semi-intensive d'élevage de porcs à Banfora, chef-lieu de la région des Cascades. Situé à $85 \mathrm{~km}$ sur l'axe Bobo-Dioulasso-Niangologo, Banfora bénéficie d'un climat e type soudanien avec une pluviométrie comprise entre $800-1200 \mathrm{~mm}$. La région des Cascades (10\% de la production nationale de mangue), les Hauts-Bassins (53\%) et le Centre Ouest $(14,1 \%)$ constituent avec les villes de Toussiana et Orodara, les principales zones de production de mangue du Burkina Faso (MASA, 2013). En plus des vergers d'où proviennent les avaries de récoltes, de nombreuses unités de production de mangue séchée et l'usine de Dafani productrice de jus installées dans les villes de l'Ouest du Burkina (Bobo-Dioulasso, Toussiana, Orodara, Bérégadougou, Banfora et Niangoloko) constituent avec les principales sources de production de sous-produits de transformation de la mangue.

L'habitat des porcs: Trois loges semi-ouvertes de $12,87 \mathrm{~m}^{2}(3,9 \mathrm{~m} \times 3,3 \mathrm{~m})$ composés d'une partie couverte de tôle et une courette chacune ont abrités les porcs. Le bâtiment est construit en banco, crépis en ciment sur les façades intérieures à hauteur de $1,35 \mathrm{~m}$. Le plancher est cimenté et comporte une légère pente pour l'évacuation du purin. Une mangeoire et un abreuvoir/loge ont été disposés pour le service du repas et de l'eau.
(Barry, 2017) au Burkina Faso renforce la nécessité des études sur la valorisation des déchets de mangue par l'alimentation des animaux. C'est en droite ligne de cette alternative en vue d'accroitre la disponibilité des aliments pour les animaux, particulièrement les porcs, qu'un procédé de production de provendes à base de d'chets de mangues a été mis au point (Kiendrébéogo et al., 2013). L'objet de l'étude a été d'évalué les effets de l'utilisation de provendes de mangue, produits par application de ce procédé, sur les performances pondérales et les caractéristiques des carcasses de porcs en croissance $(25-60 \mathrm{~kg})$ afin d'en tirer des enseignements sur leur valorisation possible par les porcs.

Les animaux: Vingt-quatre (24) porcelets de race Korhogo (12 mâles castrés et 12 femelles) de 4 mois d'âge en moyenne ont été utilisés pour le test. Ils ont été gardés en claustration permanente pendant le test. Ils ont été identifiés par des boucles auriculaires et déparasités par injection de $0,5 \mathrm{ml}$ d'ivomec $\otimes /$ porc en sous-cutanée. Ils ont enfin été pesés et regroupés en 3 lots $(1,2$ et 3$)$ de 8 porcs chacun (4 mâles et 4 femelles).

Caractéristiques des provendes et des rations : La composition chimique des provendes utilisées pour la formulation des rations présentée dans le Tableau 1 est celle de l'analyse par Kiendrébéogo et al. (2013).

Les taux d'incorporation de la peau de mangue et de la pulpe +peau dans les rations R1 et R2 ont été respectivement de $14,62 \%$ et $16,49 \%$. La composition centésimale des rations et leurs apports nutritionnels sont présentés dans le tableau2. La quantité de matière sèche (MS) distribuée par jour de chaque ration a été ajustée pour obtenir le même niveau d'énergie digestible servi qui était de $3200 \mathrm{kcal} / \mathrm{kgMS}$ au démarrage $(R 1=1285 \mathrm{~g}, R 2=1386 \mathrm{~g}$ et $R 3=1200 \mathrm{~g})$ et $4400 \mathrm{kcal} / \mathrm{kgMS}$ à partir du 45ème jour (R1=1696g, $R 2=1784 \mathrm{~g}$ et $R 3=1575 \mathrm{~g}$ ). La ration est servie en 2 repas de poids égal, le matin à $7 \mathrm{~h} 30 \mathrm{mn}$ après récupération et pesée des refus et dans l'après-midi à 16h. L'eau est servie à volonté. 
Kiendrebeogo et al., J. Appl. Biosci. 2018 Effets de rations à base de déchets de mangue sur les performances pondérales et la qualité de la carcasse de porcs Korhogo en croissance au Burkina Faso

Tableau 1 : Composition chimique et énergétique des provendes de mangue

\begin{tabular}{|c|c|c|c|}
\hline \multirow[t]{2}{*}{ Teneurs } & & \multicolumn{2}{|l|}{ Aliments } \\
\hline & & PMS & PPS \\
\hline \multicolumn{2}{|l|}{ Matières sèches } & 90,78 & 91,77 \\
\hline \multicolumn{2}{|l|}{ Matières azotées totales } & 10,61 & 8,72 \\
\hline \multicolumn{2}{|l|}{ Matières grasses } & 7,65 & 5,42 \\
\hline \multicolumn{2}{|l|}{ Cellulose brute } & 9,35 & 8,59 \\
\hline \multicolumn{2}{|l|}{ NDF } & 37,57 & 26,46 \\
\hline \multicolumn{2}{|l|}{ ADF } & 15,01 & 14,35 \\
\hline \multicolumn{2}{|l|}{ Lignine } & 4,78 & 3,34 \\
\hline \multicolumn{2}{|l|}{ Matières minérales } & 8,25 & 6,56 \\
\hline \multicolumn{2}{|l|}{ Insoluble chlorhydrique } & 4,54 & 2,2 \\
\hline \multicolumn{2}{|l|}{ Calcium } & 0,43 & 0,44 \\
\hline \multicolumn{2}{|l|}{ Phosphore } & 0,18 & 0,15 \\
\hline \multicolumn{2}{|l|}{$\mathrm{NaCl}$} & 1,23 & 0,43 \\
\hline \multicolumn{2}{|l|}{ EB (kcal/kgMS) } & 4457 & 4374 \\
\hline \multicolumn{2}{|l|}{ ED (kcal/kgMS) } & 2826 & 3204 \\
\hline \multicolumn{4}{|c|}{$\begin{array}{l}\text { NDF }=\text { Neutral Detergent Fiber } ; \text { ADF }=\text { Acid Detergent Fiber } ; E D=\text { Énergie Digestible } ; \text { PMS = Provende de peau de mangue } \\
\text { PPS = Provende de peau +pulpe de mangue }\end{array}$} \\
\hline \multicolumn{4}{|c|}{$\begin{array}{l}\text { Tableau } 2 \text { : Composition, apports nutritionnels et énergétique et coûts des rations } \\
\text { Composition centésimale réqimes alimentaires }\end{array}$} \\
\hline \multirow{2}{*}{$\begin{array}{l}\text { Composition centesimale regimes alımentair } \\
\text { Ingrédients }\end{array}$} & \multicolumn{3}{|c|}{ Régimes alimentaires } \\
\hline & R1(PPS) & R2 (PMS) & R3 (TSM) \\
\hline Son & 0 & 0 & 61 \\
\hline Provende peau+pulpe de mangue + Son (PPS) & 61 & 0 & 0 \\
\hline Provende peau de mangue+son (PMS) & 0 & 61 & 0 \\
\hline Maïs & 10 & 10 & 10 \\
\hline Drêche de dolo & 15 & 15 & 15 \\
\hline Tourteau de coton & 6,5 & 6,5 & 6,5 \\
\hline Coquillages & 1 & 1 & 1 \\
\hline Farine poisson & 5,55 & 5,55 & 5,55 \\
\hline Sel & 0,5 & 0,5 & 0,5 \\
\hline Méthionine & 0,17 & 0,17 & 0,17 \\
\hline Lysine & 0,28 & 0,28 & 0,28 \\
\hline \multicolumn{4}{|c|}{ Composition nutritionnelle des régimes alimentaires } \\
\hline & R1 & R2 & R3 \\
\hline ED (Kcal) & 2505 & 2355 & 2667 \\
\hline $\operatorname{MAB}(\%)$ & 12,54 & 13,69 & 13,62 \\
\hline Lysine (\%) & 0,62 & 0,62 & 0,8 \\
\hline Méthionine (\%) & 0,39 & 0,39 & 0,57 \\
\hline Calcium (\%) & 0,96 & 0,95 & 0,77 \\
\hline Phosphore (\%) & 0,45 & 0,47 & 0,78 \\
\hline $\mathrm{CB}(\%)$ & 6,87 & 7,34 & 4,99 \\
\hline \multicolumn{4}{|l|}{ Coûts de production des régimes alimentaires } \\
\hline Coût (FCFA) /kg MS & & 151,6 & 127,66 \\
\hline Coût (FCFA) /Énergie (kcal) & & 0,06 & 0,05 \\
\hline
\end{tabular}

PPS= Mangue entière+ son ; PMS= peau de mangue +son ; TSM=témoins sans mangue 
Collecte et analyse des données: Le suivi de l'évolution pondérale est fait par des pesées hebdomadaires avant la distribution du repas matinal. Une période d'adaptation de 2 semaines a été observée avant le début des pesées. La croissance est appréciée par l'évolution des poids et les gains moyens quotidiens (GMQ) obtenu par calcul suivant : $\boldsymbol{G M} Q(\boldsymbol{g})=\frac{(F F-F I)}{N I}$

où $\mathrm{PI}=$ poids initial, $\mathrm{PF}=$ poids final et $\mathrm{NJ}=$ durée du test (jours). Les paramètres économiques ont été appréciés à travers les indices de consommations (IC), les coûts alimentaires de production d'un (01) $\mathrm{kg}$ de viande et la qualité de la viande à travers des mesures effectuées sur la carcasse suivant les formules

(i) $I C=\frac{C A / g)}{G M Q(g)}$ avec $\mathrm{CAJ}=$ consommation alimentaire par jour obtenue par la différence entre la quantité d'aliment distribuée et le refus et $\mathrm{GMQ}=$ gain moyen quotidien défini plus haut;

(ii)

$$
\text { CAPKgV(FCFA })=\frac{\text { Coût de l'aliment total consommé }}{\text { Gain total de poids }}
$$

Pour l'évaluation de la qualité de la carcasse, 2 porcs/ Ration (01 mâle et 01 femelle) ont été prélevés, mis à jeun pendant 24 heures, pesés puis abattus à l'Abattoir Frigorifique de Bobo-Dioulasso. Après abattage le cinquième quartier a été séparé de la carcasse. La carcasse et chacun des organes (rein, foie, tête, poumons, cœur, rate, et estomac, intestin grêle, gros intestin vidés de leur contenu), ont ensuite été pesés.

\section{RESULTATS}

Teneurs en énergie digestible (ED), en MAB et en cellulose des rations: Les teneurs en énergie digestible (ED) des rations R1 et R2 ont été inférieures respectivement de $-162 \mathrm{kcal} / \mathrm{kgMS}(-6,07 \%)$ et $312 \mathrm{kcal} / \mathrm{kgMS}(-11,70 \%)$ à celle du témoin R3. Les teneurs en cellulose ont été supérieures de $+1,88 \%$ et de $+2,35 \%$ pour $\mathrm{R} 1$ et $\mathrm{R} 2$ respectifs comparées à $\mathrm{R} 3$. La teneur en MAB sensiblement égale pour R2 et R3 est plus élevée de $+1 \%$ que celle de R1. Les taux d'incorporation des déchets de mangue ont été de $14,62 \%$ (peau de mangue) et 16,49\% (peau+pulpe) dans $\mathrm{R} 1$ et $\mathrm{R} 2$ respectifs.
Une découpe a été faite suivant la méthode de Paris (Desmoulin et al., 1976). La carcasse a été fendue sur sa longueur en deux (02) parts égales suivant la ligne de la colonne vertébrale. L'épaisseur du lard sur chaque $1 / 2$ carcasse a été mesurée. La longe, la barbière, le jambon, la panne, la poitrine, ont été pesés. Pour apprécier la qualité de la carcasse, le rendement carcasse chaud (RDTc), le gras total (GT), la viande entière (VE) (muscles +os) et rapport viande entière /Gras total (VE/GT) ont été calculés selon les formules ci-après :

(i) RDTc $=\frac{(\boldsymbol{F} v-\boldsymbol{F} \boldsymbol{c})}{\boldsymbol{P} v} \boldsymbol{x} 100$ avec PV = poids vif avant l'abatage, et $\mathrm{PC}=$ poids carcasse +tête ;

(ii)

$G T=-860+1,63$ bardière $+1,43$ panne $+0,52$ poitrine avec un indice de régression $R=0,973$ (le poids des différentes partie x 1000) (Desmoulin et al., 1976);

(iii) $\boldsymbol{V} \boldsymbol{E}(\boldsymbol{k g})=\boldsymbol{P C}-\boldsymbol{G} \boldsymbol{T}$; avec $\mathrm{PC}=$ poids carcasse et $\mathrm{GT}=$ graisse totale. Nous préférons considérer les os avec les muscles car dans la pratique locale les muscles sont rarement séparés des os à la vente ; et (

iv) Le VE/GT est obtenu en divisant le poids du total de la viande (viande+os) par le poids des graisses totales (graisses de couverture et d'infiltration). Les données ont été analysées à l'aide les logiciels XLSTAT 2007 et IBM SPSS Statistics 22.

Évolution pondérale des porcs: Le poids moyens des porcs des rations R1, R2 et R3 ont été croissants du début à la fin de l'essai (figure 1). Les poids moyens initiaux (PI) de R1 $(24,8 \pm 1,7 \mathrm{~kg}), \mathrm{R} 2(25,92,9 \mathrm{~kg})$ et R3 $(25,52,4 \mathrm{~kg})$ ont été homogènes $(p>0,05)$. Ceux finaux (PF) de R1 $(49,9 \pm 4,8 \mathrm{~kg}), \mathrm{R} 2(51,5 \pm 5,8 \mathrm{~kg})$ et R3 $(56,5 \pm 8,6 \mathrm{~kg})$ ont été également homogènes $(p>0,05)$. Les poids moyens des porcs de R3 (Témoin) ont été plus élevés que ceux de R1 et R2 depuis la 2ème pesée et ceux de R2 plus élevés que R1 à partir de la 8ème pesée. 


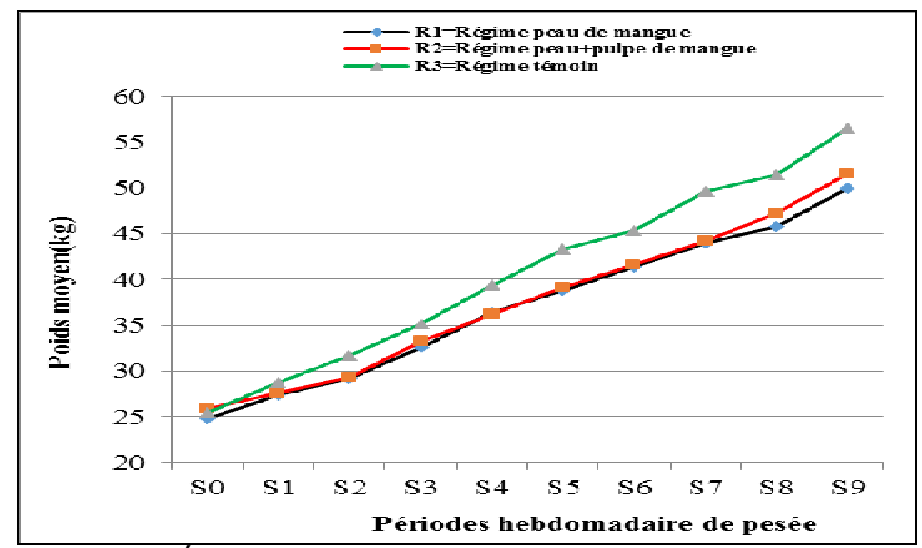

Figure 1 : Évolution pondérale des porcs en fonction des rations

Variation des GMQ, des IC et du CAPkgV des régimes alimentaires : Les résultats montrent que les $\mathrm{GMQ}$, les IC et les CAPkgV des rations $\mathrm{R} 1$ et $\mathrm{R} 2$ ont été significativement comparables (Tableau 3). Le $G M Q$, de la ration $R 3$ a été significativement $(p<0,05)$ supérieur aux GMQ des rations $\mathrm{R} 1$ et $\mathrm{R} 2$. L'IC de la ration $\mathrm{R} 3$ a été significativement inférieur à ceux des rations $\mathrm{R} 1$ et R2 (tableau 3). Le CAPkgV des trois rations ont été significativement décroissants des rations $\mathrm{R} 1, \mathrm{R} 2$ à $\mathrm{R} 3(\mathrm{p}<0,05)$.

Tableau 3: Variation des $\mathrm{GMQ}(\mathrm{g})$, IC et des CAPkgV (F CFA) observées selon les régimes

\begin{tabular}{l|l|l|l|l}
\hline Rations & N & GMQ & IC & CAPkgV \\
\hline R1 (régime peau de mangue) & 8 & $399 \pm 81^{\mathrm{a}}$ & $2,5 \pm 0,5^{\mathrm{a}}$ & $372 \pm 31^{\mathrm{a}}$ \\
\hline R2 (régime peau+pulpe de mangue) & 8 & $412 \pm 81^{\mathrm{a}}$ & $2,6 \pm 0,6^{\mathrm{a}}$ & $319 \pm 19^{\mathrm{a}}$ \\
\hline R3 (régime témoin) & 8 & $493 \pm 62^{\mathrm{b}}$ & $1,9 \pm 0,4^{\mathrm{b}}$ & $205 \pm 16^{\mathrm{b}}$ \\
\hline
\end{tabular}

* Les moyennes de la même colonne et de la même variable dépendante portant les mêmes lettres ne sont pas significativement différents $(p<0,05)$

- GMQ= gain moyen quotidien ; IC= indice de consommation ; CAPkgV= Coût alimentaire de production du kg de viande

Des différences significatives $(p<0,05)$ entre les femelles et mâles ont été observées seulement au niveau de la ration R2 (peautpulpe de mangue) (Tableau 4). Au niveau de cette ration, le GMQ des mâles a été significativement supérieur à celui des femelles; l'IC des femelles a été significativement supérieur à celui des mâles; le CAPkgV des femelles a été significativement inférieur $(P<0,05)$ à celui des mâles.

Tableau 4 : Variation des GMQ (g), de l'IC et du CAPkgV (F CFA) suivant les régimes et le sexe

\begin{tabular}{l|c|c|c|c|c|c|c|c|c}
\hline \multirow{2}{*}{ Variables } & \multicolumn{9}{|c|}{ Régimes alimentaires } \\
\cline { 2 - 11 } & \multicolumn{3}{|c|}{ R1(PMS) } & \multicolumn{3}{c|}{ R2 (PPS) } & \multicolumn{3}{c}{ R3 (TSM) } \\
\hline Sexe & $\begin{array}{c}\text { Femelle } \\
(\mathrm{n}=4)\end{array}$ & Mâle $(\mathrm{n}=4)$ & Sig. & $\begin{array}{c}\text { Femelle } \\
(\mathrm{n}=4)\end{array}$ & Mâle $(\mathrm{n}=4)$ & Sig. & $\begin{array}{c}\text { Femelle } \\
(\mathrm{n}=4)\end{array}$ & $\begin{array}{c}\text { Mâle } \\
(\mathrm{n}=4)\end{array}$ & Sig. \\
\hline $\mathbf{G M Q ( g )}$ & $\begin{array}{c}396,4 \pm 58, \\
9\end{array}$ & $400,8 \pm 107,9$ & 0,946 & $\begin{array}{c}346,4 \pm 53 \\
, 1 \mathrm{a}\end{array}$ & $\begin{array}{c}477,4 \pm 33,2 \\
\mathrm{~b}\end{array}$ & 0,000 & $\begin{array}{c}460,3 \pm 10 \\
7,5\end{array}$ & $\begin{array}{c}525 \pm 104, \\
5\end{array}$ & $\begin{array}{c}0,42 \\
1\end{array}$ \\
\hline IC & $2,46 \pm 0,36$ & $2,53 \pm 0,69$ & 0,86 & $3 \pm 0,51 \mathrm{a}$ & $2,14 \pm 0,15 \mathrm{~b}$ & 0,020 & $2 \pm 0,5$ & $1,8 \pm 0,4$ & 0,41 \\
\hline $\begin{array}{l}\text { CAPkgV } \\
\text { (FCFA) }\end{array}$ & $372 \pm 55$ & $372 \pm 120$ & 0,998 & $364 \pm 32 \mathrm{a}$ & $274 \pm 19 \mathrm{~b}$ & 0,003 & $219 \pm 52$ & $190 \pm 403$ & 0,41 \\
\end{tabular}

${ }^{*}$ Les moyennes de la même ration sur la même ligne portant les mêmes lettres ne sont pas significativement différents $(p<0,05)$

GMQ= gain moyen quotidien ; IC = indice de consommation ; CAPkgV= Coût de production du $\mathrm{kg}$ de viande ;

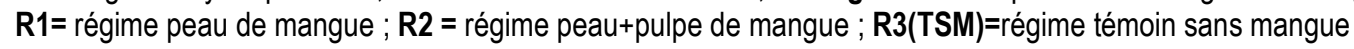


Caractéristiques de la carcasse: L'analyse de variance sur les poids des différentes parties de la carcasse et du 5ème quartier, n'ont pas montré de différences significatives $(p<0,05)$ entre les Rations pour le rendement carcasse (RDTc), la proportion de gras total (GT) et de viande entière (VE) et du rapport viande entière sur gras total (VE/GT) (tableau 4). En valeur absolue, le RDTc, la proportion de VE et l'indice VE/GT ont été plus élevés pour les rations R1 (peau de mangue) et R2 (peau + pulpe de mangue) que ceux de la ration témoin R3. La proportion de GT a été plus élevée dans la ration R3 que dans les rations $\mathrm{R} 1$ et $\mathrm{R} 2$ (tableau 5).

Tableau5 : Rendement carcasse (\%), Proportion (\%) de gras et de viande et rapport Viande T/graisse suivant le type de ration et le sexe

\begin{tabular}{|c|c|c|c|c|c|}
\hline Rations & Sexe & $\begin{array}{c}\text { Rendement } \\
\text { carcasse (RC)) }\end{array}$ & $\begin{array}{c}\text { Graisses totales } \\
\text { (GT) }\end{array}$ & $\begin{array}{l}\text { Viande entière } \\
\text { (VE) }\end{array}$ & VT/GT \\
\hline \multirow{3}{*}{ Ration1 (n=2) } & Femelle & 79,2 & 30.3 & 69.7 & 2.3 \\
\hline & Mâle & 87,5 & 31,1 & 68,9 & 2,2 \\
\hline & lot1 & $83,4 \pm 5,9^{a}$ & $30,7 \pm 0,6^{a}$ & $69,3 \pm 0,6^{a}$ & $2,3 \pm 0,1^{a}$ \\
\hline \multirow{3}{*}{ Ration 2 ( $\mathrm{n}=2$ ) } & Femelle & 77,6 & 29,5 & 70,5 & 2,4 \\
\hline & Mâle & 77,1 & 31,7 & 68,3 & 2,2 \\
\hline & lot2 & $77,3 \pm 0,4^{a}$ & $30,6 \pm 1,6^{a}$ & $69,4 \pm 1,6^{a}$ & $2,3 \pm 0,2^{\mathrm{a}}$ \\
\hline \multirow{3}{*}{ Ration3 (n=2) } & Femelle & 75,9 & 31,5 & 68,5 & 2,2 \\
\hline & Mâle & 76,6 & 34,2 & 65,8 & 1,9 \\
\hline & lot3 & $76,2 \pm 0,5^{a}$ & $32,8 \pm 1,9^{a}$ & $67,2 \pm 1,9^{a}$ & $2,1 \pm 0,2^{a}$ \\
\hline
\end{tabular}

- Les moyennes de la même colonne portant les mêmes lettres ne sont pas significativement différentes $(p>0,05)$

Ration1= régime peau de mangue ; Ration2= régime peau+pulpe de mangue ; Ration3=régime témoin

\section{DISCUSSION}

Performances de croissance : Les GMQs des porcs nourris avec les rations $R 1$ et $R 2$, sont significativement inférieures à celle des porcs du témoin $\mathrm{R} 3(p<0,05)$; dans le même temps l'évolution pondérale a été continue dans toutes les rations. La faible concentration énergétique de ces rations due à la nature fibreuse des provendes qu'elles incorporent, comparativement à R3, pourrait expliquer cette situation. Kennelly et Aherne (1980) rapportent que des taux élevés de cellulose dans des rations de porcs en croissance de $22-92 \mathrm{~kg}$ n'ont eu aucun effet dépressif sur la croissance. Ceci pourrait expliquer que malgré les taux plus élevé de $C B$ dans les rations $\mathrm{R} 1$ et $\mathrm{R} 2$ l'évolution pondérale des porcs n'ait pas subit une inflexion au cours de l'essai. Toutefois, Agyekum et Nyachoti. (2017) rapportent qu'un apport de $7 \%$ de cellulose dans la ration de porcs en croissance réduit les gains de poids et le poids final des animaux et on comprend bien pourquoi les $\mathrm{GMQ}$ de R1 et R2 aient été significativement inférieurs à celui de R3. Cette situation est certainement aggravée par la lignine, totalement indigeste, contenue dans les provendes de mangues (tableau 1). Un taux élevé de cellulose entraine la baisse de la concentration énergétique, une augmentation de la consommation d'aliment, de l'indice de consommation et une réduction de la vitesse d'ingestion (Levasseur et al., 1998; Chabeauti et Noblet, 1990) et par conséquent la consommation d'énergie (Zang et al., 2013). Les GMQ des rations R1 (468 g) et R 2 (481 g) sont proches des $488 \mathrm{~g}$ rapporté par Alfons et al. (2004) pour des porcs de races Basques. Ils sont également proches du GMQ de 482g rapporté par Déprés et al. (1992) pour des porcs de race créoles de 6 mois d'âge. Tous ces GMQ sont supérieurs à ceux rapportés par BougoumaYamoégo et al. (2005) pour des porcs de race locale ayant reçu des rations moyenne (3500 kcal/kg MS), hyper énergétique $(3700 \mathrm{kcal} / \mathrm{kg} \mathrm{MS})$ et hypo énergétique $(2800 \mathrm{kcal} / \mathrm{kg} \mathrm{MS})$. La faible teneur en CB a pu justifier une digestibilité plus élevée de la ration témoin R3 comparativement aux rations R1 et R2. La même tendance est rapportée par Goff et al. (2002) pour un premier régime titrant moins de fibres $\mathrm{CB}(0,70$ fois,) $\operatorname{ADF}(0,84)$ et $\operatorname{NDF}(0,82)$ comparativement à un deuxième régime distribué à des porcs en croissance. Henry (1969) recommande des niveaux de cellulose de l'ordre de $8 \%$ pour les produits pulvérulents et de $5 \%$ pour les produits à texture fibreuse. La formulation des rations incorporant les provendes de mangue pourrait tenir compte de ces recommandations. 
Performances techniques et économique des rations : L'examen des IC montre que la ration témoin R3 a été mieux valorisée, suivie de la ration R2 dont I'IC $(3,95)$ est proche de celui de 3,85 rapporté par Bosma et al. (2004) pour des porcs de même race précocement engraissés. La plus grande teneur en fibre de R1(PMS) $(+0,06 \%)$ et R2 (PPS) $(+0,10 \%)$ explique cette situation. En effet Meffeja et al. (2007) rapportent des résultats similaires qui démontrent que la dilution énergétique de rations par une substitution élevée (75 et $100 \%$ ) du tourteau de coton par du tourteau de palmiste avait eu pour conséquence des IC plus élevés et des carcasses moins grasses comparativement aux rations témoins moins concentrées en fibre (25\% et $50 \%)$. L'action stimulante de la cellulose sur la consommation de nourriture est surtout nette pendant la première phase de la croissance entre 20 et $60 \mathrm{~kg}$ de poids vif comme c'est le cas dans notre expérimentation. Dans le même sens des rations diluées par des niveaux d'incorporation croissante de coques de cacao $(10 \%, 20 \%$ et $30 \%$ ) ont eu pour conséquence, en plus d'indices de consommation plus élevés que ceux de la ration témoin, la réduction de l'épaisseur du lard dorsale (Meffeja et al., 2006). Par conséquent, ces régimes réduisent la vitesse de croissance (Levasseur et al., 1998). Au niveau de substitution par $61 \%$ des provendes PMS et PPS dans chacune des rations R1 et R2 au son de maïs dans la R3, le dE plus élevé du PPS (73\%) a certainement compensé la faible disponibilité de l'Énergie causée par sa concentration plus élevée en fibre que le son de maïs ( $\mathrm{dE}=59 \%$ ). Ceci explique les valeurs proches de vitesse de croissance et à terme des IC des deux régimes expérimentaux. L'on peut comprendre par ailleurs que les carcasses

\section{CONCLUSION}

L'étude a permis de mesurer les performances pondérales et quelques caractéristiques des carcasses de porcs de race Korhogo nourris avec des rations incorporant des provendes (PMS et PPS) de mangue. En dépit des GMQs et des poids finaux des rations $\mathrm{R} 1$ et $R 2$ moins élevés que ceux de la ration témoin $R 3$, les IC, les rapports coûts/énergie des rations quasiment égaux pour les trois Rations ainsi que le rapport Viande entière / Gras total plus élevé dans les rations R1 et R2 montrent que ces dernières ont été mieux valorisées. Les provendes pourront bien se substituer au son de maïs voir au maïs qui était en faible proportion dans les aient été plus grasses pour les porcs du régime témoin R3 d'abord, puis R1 et enfin R2 conformément à l'hypothèse de Kiendrébéogo et al. (2013) selon laquelle les provendes PMS et PPS prédisposeraient les régimes qui les incorporent à la production de viande maigre. Ce type de viande se prête mieux aux habitudes culinaires africaines notamment la viande cuite au four, frite, braisée et les brochettes en général consommée hors-foyer (Centres, 1996; Mopaté et al., 2006; Kiendrébeogo et al., 2012b) et à la demande des consommateurs asiatiques (Wuntu, 1993; Berger et Pham, 1997 ; Patricot, 2002). Le CAPkgV de R3 est moins cher que ceux des rations R1 et R2. Le niveau quasiment égal des coûts de l'offre énergétique, les meilleurs RDMTc VE/GT, la bonne qualité de la viande (maigre) de R1 et R2 compensent largement cette infériorité du CAPkgV de R3. Le RDMTc de R1 a été supérieur aux rendements carcasses de deux rations dites hyper et hypo énergétique rapportés par Bougouma-Yamoégo et al. (2005). L'indice VE/GT a été plus élevé de + de 0,67 points que ceux rapportés par les mêmes auteurs. II apparait clairement qu'il est préférable d'avoir recours aux régimes incorporant les provendes qui permettent un bon rendement et la production de viande maigre par rapport à la ration témoin, d'autant plus que les viandes maigres sont plus recherchées et que la graisse n'est pas particulièrement valorisée. Ces aliments peuvent être spécifiquement recommandés pour la croissance des mâles. En effet ces derniers valorisent mieux les rations à base de déchets de mangues comme l'indique les IC et les coûts alimentaires de production (CAPkgV) moins élevés comparativement aux femelles (tableau 4). Les déchets de mangues constituent une alternative à une plus grande disponibilité des aliments pour les porcs.

rations. Cette alternative permet d'augmenter la disponibilité des aliments pour les porcs et les autres animaux d'élevage. Elle permet également la production e viande maigre couplée à de bons RDMTc, ce compense le coût plus élevé du CAPkgV des rations $R 1$ et R2 que celui de R3. Toutefois il est nécessaire de poursuivre les recherches en vue de produire des provendes moins chères avec pour conséquence de faire baisser le coût de production du $\mathrm{kg}$ de viande. De même, des études de digestibilité des provendes et des rations les incorporant seront nécessaires pour affiner les recommandations de leur utilisation. 


\section{REMERCIEMENTS}

Nous remercions, monsieur Yaguibou François, Agent d'élevage retraité à Banfora qui a offert gracieusement ses installations pour la conduite de l'essai, Mr. Koné

\section{BIBLIOGRAPHIE}

Agyekum A.K., Nyachoti C. M., 2017. Nutritional and Metabolic consequences of feeding High-Fiber Diets to Swine: A review. Animal Nutrition and feed Science -Review, Engineering 3 (2017) 716-725.

Alfons L., Mourot J., Insausti K., Mendizabal J. A., Arana A., 2004. Comparative description of growth, fat deposition, carcass and meat quality characteristics of Basque and Large White pigs. Animal Research, 54 (2005) 3342, ( ) INRA, EDP Sciences, 2005

Barry D., 2017. Effets de régimes alimentaires à base de mangue sur la croissance et les paramètres biométriques des œufs de poules pondeuses au Burkina Faso. Mémoire d'Ingénieur de Développement Rural, Université de Dédougou, Dédougou, Burkina Faso, 45p.

Bergeret P., Pham Hoang Ha, 1997. Dynamiques comparés de trois filières dans le delta du fleuve Rouge: riz, porc, ail. Cahiers Agricultures, 6: 337 - 343, Agriculture et développement, 15: 19 - 26

Bosma R H, ZongoL C, Sané A., Chantal Zoungrana et Soudré A., 2004. Comparaison participatoire de trois méthodes d'engraissement des porcs dans les provinces du Sanguié et du Boulkiemdé au Burkina Faso. Livestock Research for Rural Development, Vol. 16, Art. \#10. Retrieved February 11, 2013, from http://www.Irrd.org//rrd16/2/bosm1602.htm

Bougouma-Yamoégo V. M. C., Ouédraogo C. L., Wereme-Ndiaye A. et Konkobo B., 2005. Influence du niveau énergétique sur les performances de croissance et d'engraissement bouchères du porc local: valeur bouchère et rentabilité économique des rations. Article original in RASPA, Vol $3 \mathrm{~N}^{\circ} 3-$ 4., 2005.

Chabeauti E., Noblet J., 1990. Digestion par le porc de quatre sources de parois végétales utilisées seules ou en association. Journée de Recherches porcines en France, 22, 167-174.

Centres J. M., 1996. L'élevage et l'agriculture en zones urbaines et périurbaines dans deux villes de la Ferme KONA de Banfora qui nous a permis d'utiliser son broyeurs mélangeur pour la fabrication des rations.

sahéliennes: Bamako et Bobo-Dioulasso. Cahiers Agricultures, 5 (5) : $373-381$

Déprés E., Tamisier F., Naves M., Rinaldo D., 1992. Comparaison de porcs Créoles et large White pour les performances de croissance et la qualité de la viande en fonction de l'âge d'abattage. Journées de recherches porcines en France, 24, 17-24. http://www.journeesrecherche-

porcine.com/texte/1992/92txtGenetique/G9203 .pdf (28/01/2012)

Desmoulin B., Grandsart P. et Tassencourt L., 1976. Les critères d'appréciation de la composition anatomique de la carcasse du porc et des pièces de découpe. In Journées de recherches porcines en France, 1976, 89-98. http//journées-recherche-

porcine.com/texte/1976/76txtCarcasse/C7601 pdf (28/01/2012)

FAO (Organisation des Nations Unies pour l'Alimentation et l'Agriculture), 2018. Consommation de viande. Département de l'agriculture et de la protection des consommateurs, Production et Santé animale. http://www.fao.org/ag/againfo/themes/fr/meat/b ackground.html (10/05/2018)

FAOSTAT, 2015. () OAA Division de la Statistique 2015. Faostat.org/site/569 (19 novembre 2015).

Goff G. L., Dubois S., Milgen J. V, Noblet J. 2002. Influence of dietary fiber level on digestive and metabolic utilization of energy in growing and finishing pigs. Anim. Res. 51 (2002) 245-259

Kennelly, J. J., et Aherne F. X., 1980. The effect of fiber addition to diets formulated to contain different levels of energy and protein on growth and carcass quality of swine. Canadian Journal of Animal Science 60 (2): 385-393. http://www.nrcresearchpress.com/doi/abs/10.4 141/cjas80-048\#.W6IsmM77TIU (17/09/ 2018

Kiendrébéogo T., 2006. Diagnostic des élevages porcins de la zone de Bobo-Dioulasso: Systèmes d'élevage et conditions technicoéconomique de production. Mémoire de DEA. 
Université polytechnique de Bobo-Dioulasso, Burkina Faso. 55p.

Kiendrébéogo T., Hamadou S. Mopaté L., KaboréZoungrana C-Y., 2008. Typologie des élevages porcins urbains et périurbains de Bobo-Dioulasso (Burkina Faso). Revue Africaine de Santé et de Productions Animales (RASPA) Vol.6 N03-4, 2008

Kiendrébéogo A., Mopaté L. Y., Kondombo S. R., Kaboré-Zoungrana C. Y. 2012a. Characterization and importance of pig breeds in the pork industry of the zone of BoboDioulasso (Burkina Faso, West Africa). Int. J. Biol. Chem. Sci. 6 (4): 1535-1547,

Kiendrébeogo T., Mopaté Logténé Y., Kondombo S. R., Kaboré-Zoungrana C. Y., 2012b. Approvisionnement en porcs vifs et viande porcine de la ville de Bobo-Dioulasso (Burkina Faso). Journal of Agriculture and Environment for International Development (JAEID), 106 (2) : 105-122

Kiendrébéogo T., Mopaté Logténé Y., Ido G., KaboréZoungrana C. Y., 2013. Procédés de production d'aliments non-conventionnels pour porcs à base de déchets de mangues et détermination de leurs valeurs alimentaires au Burkina Faso. Journal of Applied Biosciences (JAB) 67: 5261-5270.

Kiendrébéogo T., Mopaté Logténé Y., Ido G., KaboréZoungrana C. Y., 2014. The Typology of the pig breeding in Burkina Faso: cases of the towns of Bobo-Dioulasso and Gaoua in the soudanian area; Kaya and Dori in the sahelian area. International Journal of Agronomy and Agricultural Research (IJAAR), Vol.4, N5, p. 119-136, 2014.

Levasseur P., Corboulay V., Meunier-Salaün M-C., Bourmad J-Y, Noblet J., 1998. Influence de la source d'énergie et de la concentration énergétique de l'aliment sur le comportement alimentaire, les performances zootechniques et les qualités de carcasse du porc charcutier. Journée de Rech. Porcine en France, 30, 245252. http://journees-recherche-porcine.com/ texte/1998/98alim/al9809.pdf (10/10/2015)

MASA (Ministère de l'agriculture et de la Sécurité Alimentaire), 2013. Situation de référence des principales filières agricoles au Burkina Faso. Rapport version finale. http://www.uncdf.org/sites/default/files/downloa $\underline{\mathrm{d} / \text { rapport final situation de reference filieres }}$ agricoles.pdf

Meffeja F., Dogmo T., Njifutie N. and Tchakounté J. 2007 : Influence de la substitution du tourteau de coton par le tourteau de palmiste dans l'alimentation des porcs en croissance finition. Livestock Research for Rural Development. Volume 19, Article \#18. Retrieved September 18, 2018, from http://www.llrd.org//rrd19/2/meff19018.htm (18 septembre 2018)

Meffeja F., Njifutié N., Manjeli Y., Dongmo T., Tchakounté J. et Fombad R. B. 2006 : Digestibilité et influence des rations contenant des niveaux croissants de coques de cacao sur les performances des porcs en croissance finition. Livestock Research for Rural Development. Volume 18, Article No. 165. Retrieved September 18, 2018, from http://www.llrrd.org//rrd18/11/meff18165.htm (18 septembre 2018)

Mankor A., 2009. Consommation urbaine de viande en Afrique de l'Ouest : l'exemple de Dakar. Article in Le Dossier Évolution du secteur de l'élevage Ouest Africain ; in Grain de sel N46-47, p. 1617, mars-août 2009.

MED/MRA, 2004. Deuxième enquête nationale sur les effectifs du cheptel, Tome II, Résultats et analyses (Version finale). 77p.

MRA (Ministère des Ressources Animales), 2015. Annuaire des statistiques de l'élevage 2014. Rapport annuel sur les statistiques de l'élevage au Burkina Faso. 177p. http://cns.bf//MG/pdf/annuaire_2014_mra_vf.p df

Mopaté L. Y., Koussou M. O., Kaboré-Zoungrana C. Y., Gouro A., 2006. Commerce et consommation de la viande porcine dans la zone de N'Djaména (Tchad). Revue Sénégalaise de recherches Agricoles et agroalimentaires (RSRAA), 1 (2) : $39-48$

Mopaté L. Y., Kaboré -Zoungrana C-Y., 2011. Disponibilité et valeurs alimentaires des sons de riz, maïs et sorgho mobilisables dans l'alimentation des porcs à N'Djaména (Tchad). Journal of Applied Biosiences (JAB), 2757 $2764 p$.

Noblet J., Henry Y., Bourdon D., Blondel Ch., Calmes R., et al.., 1980. Influence d'une réduction du taux d'azote indifférencié sur le niveau d'ingestion alimentaire et les performances de 
croissance du porc femelle, selon la concentration et la nature des substrats énergétiques dans le régime. Annales de zootechnie, 1980, 29 (2), pp.103-119. <hal00887948>

Patricot S., 2002. Les systèmes d'élevages familiaux de porcs au Nord-Viêtnam. Synthèse bibliographique. DESS Productions animales en régions chaudes. Année Universitaire, 2001 - 2002, Cirad-Emvt, Montpellier, France, 38 p. + annexes $21 \mathrm{p}$.

Sawadogo C., 2016. Effet de l'incorporation d'aliments non conventionnels à base de déchets de mangue dans la ration des poulets (Gallus gallus domesticus) de race locale en croissance-finition au Burkina Faso. Mémoire de fin de cycle d'Ingénieur de Développement rural, Université de Dédougou, 39p.

UEMOA (Union Économique et Monétaire Ouest Africaine), 2014. Rapport annuel sur la surveillance commerciale de I'UEMOA. 79p. http://www.uemoa.int/sites/default/files/biblioth eque/rapport_2013_de_la_surveillance_comm erciale version finale 0.pdf

Wuntu Norrytha Lineke, 1993. Étude du rôle des fibres dans l'alimentation porcine. Synthèse bibliographique. DESS Productions animales en régions chaudes. Année universitaire 19921993, Cirad-Emvt, Maisons-Alfort, France, 12 p.

Zhang W., Li D., Zhang J., Duan Q., Yang W. and Zhang L., 2013. The effects of dietary fiber level on nutrient digestibility in growing pigs. Journal of Animal Science and Biotechnology, 2013, 4: 17 http//www.jasbsi.com/content $\underline{14 / 1 / 17}$ 